\title{
Tightening simple mixed-integer sets with guaranteed bounds
}

\author{
Daniel Bienstock and Benjamin McClosky \\ Columbia University \\ New York
}

July 2008

\begin{abstract}
In this paper we study how to reformulate knapsack sets and simple mixed integer sets in order to obtain provably tight, polynomially large formulations.
\end{abstract}

\section{Introduction}

In this paper we consider $0 / 1$ knapsack sets and certain simple fixed-charge network flow sets. The study of such sets is relevant in that a popular approach for solving general mixed-integer programs consists of selecting a subset of constraints with particular structure (such as a single-node fixedcharge flow problem) and tightening that part of the formulation through the use, for example, of classical cutting-plane families (see e.g. [14], [11]). A question of interest is in what sense the resulting stronger formulation is provably good.

Motivated by questions posed in [17], and extending the study initiated in [3], we show how the use of appropriate disjunctions [1] leads to provably tight, yet polynomially large, formulations for several simple sets. Unlike the use of familiar disjunctive cuts ([2], [16] and [9], [10]) the disjunctions we employ are 'combinatorial', or 'structural', that is to say, they depend on the structure of the problem at hand. Previous work [6] has shown how structural disjunctions can lead to provably good approximations of combinatorial polyhedra (also see [12], [4], [5]); we expect that many other results of this type are possible.

\subsubsection{Minimum knapsack}

In Section 2 we consider the "minimum" 0/1 knapsack problem,

$$
\begin{aligned}
& (\mathcal{K} \mathcal{M} \mathcal{I} \mathcal{N}): \quad v^{Z}=\min \sum_{j=1}^{N} c_{j} x_{j} \\
& \text { s.t. } \quad \sum_{j=1}^{N} w_{j} x_{j} \geq b, \\
& x \in\{0,1\}^{N},
\end{aligned}
$$

where $c_{j}>0$ and $0<w_{j} \leq b$ for $1 \leq j \leq N$. We denote by $v^{*}$ the value of the LP relaxation of $\mathcal{K} \mathcal{M} \mathcal{I N}$.

In [8], Carr, Fleischer, Leung and Phillips consider the so-called knapsack-cover inequalities, which are different from cover inequalities. Given a set $A \subseteq\{1, \ldots, N\}$ write $b(A)=b-\sum_{j \in A} w_{j}$. Then the knapsack-cover inequality corresponding to $A$ is

$$
\sum_{j \notin A} \min \left\{w_{j}, b(A)\right\} x_{j} \geq b(A) .
$$


In [8] it is shown that the relaxation to $\mathcal{K} \mathcal{M} \mathcal{I N}$ provided by all knapsack-covers yields a lower bound $\hat{v}$ to $v^{Z}$ such that $v^{Z} \leq 2 \hat{v}$. On the other hand, the price to be paid is that the algorithm used to (approximately) solve this relaxation is fairly complex - a separation procedure for knapsack-covers is not given.

We show how a simple disjunction provides a polynomially large linear programming relaxation to $\mathcal{K} \mathcal{M} \mathcal{I} \mathcal{N}$ whose value $\bar{v}$ satisfies $v^{Z}<2 \bar{v}$. In fact, we show

Theorem 1.1 For each $0<\epsilon<1$ there is a linear programming relaxation to $\mathcal{K} \mathcal{M} \mathcal{I} \mathcal{N}$ with $O\left((1 / \epsilon)^{O\left(1 / \epsilon^{2}\right)} N^{2}\right)$ variables and constraints, whose value $v(\epsilon)$ satisfies $v^{Z}<(1+\epsilon) v(\epsilon)$.

Recently, Carnes and Shmoys [7] presented a primal-dual, 2-approximation algorithm for $\mathcal{K} \mathcal{M I \mathcal { N }}$ that relies on knapsack-cover inequalities. The constructions underlying Theorem 1.1 can be used to tighten the approximation factor to $(1+\epsilon)$ for arbitrary $0<\epsilon<1$; as far as we know, this is the first such result for $\mathcal{K} \mathcal{M} \mathcal{I} \mathcal{N}$.

\subsubsection{Fixed-charge sets}

In Section 3 we consider the fixed-charge network flow problem. We consider the following generalization given by the following mixed-integer program:

$$
\begin{aligned}
(\mathcal{F} \mathcal{X N}): \quad v^{Z}= & \min \sum_{(i, j) \in \mathcal{A}}\left(f_{i j} y_{i j}+c_{i j} x_{i j}\right), \\
& \sum_{(i, j) \in \delta^{+}(i)} x_{i j}-\sum_{(j, i) \in \delta^{-}(i)} x_{j i}=b_{i}, \quad \forall i \in \mathcal{I}, \\
& 0 \leq x_{i j} \leq u_{i j} y_{i j} \quad \forall(i, j) \in \mathcal{A}, \\
& y_{i j}=0 \text { or } 1, \quad \forall(i, j) \in \mathcal{A} .
\end{aligned}
$$

Here,

- We are given a directed graph $\mathcal{G}$,

- $\mathcal{A}$ is the set of arcs; for each $(i, j) \in \mathcal{A}$ we have $c_{i j}, f_{i j}$ nonnegative and $u_{i j}>0$,

- The vertex set of $\mathcal{G}$ is partitioned into two classes: $\mathcal{I}$ (the "inner" vertices) and $\mathcal{O}$ (the "outer" vertices),

- For each $i \in \mathcal{I}$ there is a real $b_{i} ; \delta^{+}(i)$ (resp., $\delta^{-}(i)$ ) is the set of $\operatorname{arcs}$ of the form $(i, j)$ (resp., $(j, i))$,

- Each vertex $v \in \mathcal{O}$ is incident with a unique arc; the other endpoint of that arc is an inner vertex.

In this paper we consider the special case of this problem where the inner vertices induce a path; see Figure 1. In this case we have a generalization of the single-item lot-sizing problem. The following result holds:

Theorem 1.2 Assuming $\mathcal{I}$ induces a path, then for each $0<\epsilon<1$ there is a linear programming relaxation to $\mathcal{F} \mathcal{X N}$ with $O\left((1 / \epsilon)^{O\left(1 / \epsilon^{2}\right)}(1+|\mathcal{O}|)^{3}|\mathcal{I}|^{3}\right)$ variables and constraints, whose value $v(\epsilon)$ satisfies $v^{Z}<(1+\epsilon) v(\epsilon)$. 


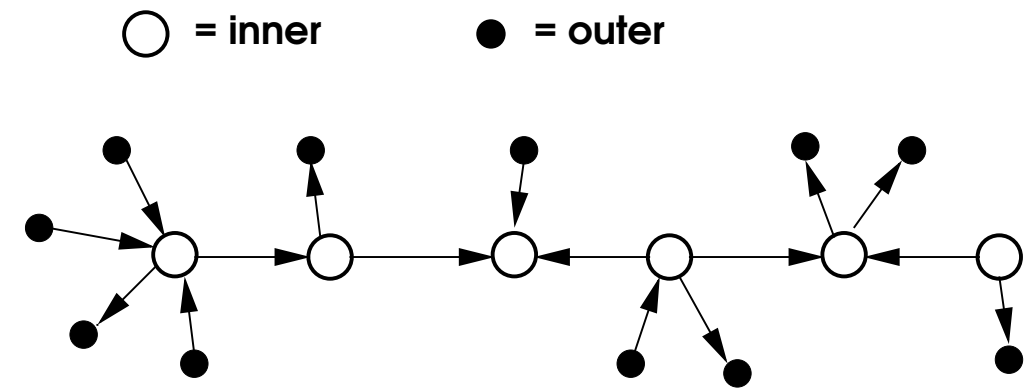

Figure 1: Fixed-charge set.

Carnes and Shmoys [7] also present a 2-approximation algorithm for the single-item lot-sizing problem; our constructions can be used to tighten their results to obtain $1+\epsilon$-approximation algorithms.

\subsubsection{Maximum knapsack}

In Section 4 we consider the "maximum" 0/1 knapsack problem,

$$
\begin{aligned}
& (\mathcal{K} \mathcal{M} \mathcal{A} \mathcal{X}): \quad v^{Z}=\max \sum_{j=1}^{N} p_{j} x_{j}, \\
& \text { s.t. } \quad \sum_{j=1}^{N} w_{j} x_{j} \leq b, \\
& x \in\{0,1\}^{N},
\end{aligned}
$$

where $p_{j}>0$ and $0<w_{j} \leq b$ for $1 \leq j \leq N$. We denote by $v^{*}$ the value of the LP relaxation of $\mathcal{K} \mathcal{M} \mathcal{A X}$.

In [17] Van Vyve and Wolsey ask whether, given an instance of $\mathcal{K} \mathcal{M} \mathcal{A} \mathcal{X}$, and $0<\epsilon \leq 1$, there is a formulation of the form $A x+A^{\prime} x^{\prime} \leq b^{\prime}$, such that

(a) For each vector $x \in\{0,1\}^{n}$ with $\sum_{j=1}^{n} w_{j} x_{j} \leq b$ there exists $x^{\prime}$ such that $A x+A^{\prime} x^{\prime} \leq b^{\prime}$,

(b) The number of variables $x^{\prime}$ and rows of $A$ and $A^{\prime}$ is polynomial in $n$ and/or $\epsilon^{-1}$, and

(c) For every $w \in R_{+}^{n}$,

$$
\max \left\{w^{T} x: A x+A^{\prime} x^{\prime} \leq b^{\prime}\right\} \leq(1+\epsilon) v^{Z}
$$

In [3] we provided a partial answer to this question: there $i s$ a formulation satisfying (a)-(c) which has polynomially many variables and constraints for each fixed $\epsilon$. This formulation amounts to a multi-term disjunction, which, although polynomial, is complex from a practical perspective.

The result in [3] motivates several questions, in particular:

1. Is there a formulation achieving (a)-(c) but restricted to the original space of variables?

2. How about achieving (c), but restricting to the original space of variables and allowing exponentially many constraints, so long as these are polynomially separable? 
3. In fact, what can be achieved in polynomial time? Assuming $w_{j} \leq b$ for all $j$, it is known that $v^{*} / v^{Z}<2$, and that this bound is best possible. Is there a "simple" relaxation involving polynomially separable inequalities, whose value $\hat{v}$ satisfies $\hat{v} / v^{Z}<\theta$ for some $\theta<2$ ?

Concerning (a), a natural question to ask is the following. Suppose we pick a fixed integer $k>0$, and we strengthen the LP relaxation of $\mathcal{K} \mathcal{M} \mathcal{A} \mathcal{X}$ with all valid inequalities of the form $\sum_{j} \alpha_{j} x_{j} \leq \beta$, where the $\alpha_{j}$ take values in $\{0,1, \ldots, k\}$. Is it true that the value of the resulting linear program is at most

$$
(1+f(1 / k)) v^{Z}
$$

where $f(\epsilon) \rightarrow 0$ as $\epsilon \rightarrow 0$ ? The answer to this question is (perhaps, not surprisingly) negative. We show that for each $k>0$, if $N$ is large enough there is an example of $\mathcal{K} \mathcal{M} \mathcal{A} \mathcal{X}$ where, after adding all valid inequalities with left-hand coefficients in $\{0,1, \ldots, k\}$, the value of linear program remains arbitrarily close to $2 v^{Z}$. This is discussed in Section 4.1. Thus, valid inequalities with "small" coefficients are not enough to get an LP to IP ratio strictly bounded away from 2 . At the same time, in Section 4.2 we show that using a (polynomially separable) disjunction, one obtains

a relaxation whose value is at most $\left(1+\frac{\sqrt{19}-2}{3}\right) v^{Z}$. Thus, question 3 above does have a positive answer.

However, the disjunction used in Section 4.2 depends on the structure of the objective coefficients $p_{j}$ and is therefore not quite in the "a priori strengthening" spirit of the question of Van Vyve and Wolsey. Further, the examples in Section 4.1 have "large" constraint coefficients, that is to say we have $w_{j} \approx b$ for some $j$. One might consider such examples "artificial" and wonder what happens when they are excluded.

These issues are taken up in Section 4.3. Given a subset $S \subseteq\{1,2, \ldots, N\}$ with $w_{i}+w_{j}>b$ for each pair of distinct indices $i, j \in S$, the clique inequality [13]

$$
\sum_{j \in S} x_{j} \leq 1
$$

is valid for $\mathcal{K} \mathcal{M A X}$. Let $v^{\omega}$ denote the value of the linear program obtained by augmenting the continuous relaxation of $\mathcal{K} \mathcal{M} \mathcal{A X}$ with all clique inequalities. In Section 4.3 we prove:

Theorem 1.3 For each constant $0 \leq \lambda<1$ there exists $\epsilon=\epsilon(\lambda)>0$ satisfying the following property. For $N$ large enough, if $w_{j} \leq \lambda b$ for $1 \leq j \leq N$, then $v^{\omega} \leq(2-\epsilon) v^{Z}$.

\section{Minimum knapsack problem}

In this section we consider problem $\mathcal{K} \mathcal{M} \mathcal{I} \mathcal{N}$ and prove Theorem 1.1. Our construction is inspired by that in [18], [5] in the context of the set-covering problem, and it relies on disjunctive inequalities. Prior to our main proof, we will first show a simpler result in order to motivate our approach. In what follows we will assume without loss of generality that

$$
c_{1} \geq c_{2} \geq \ldots \geq c_{N} .
$$

For $1 \leq h \leq N$, let $P^{h}$ denote the polyhedron defined by:

$$
\begin{aligned}
& \sum_{j=1}^{N} w_{j} x_{j} \geq b, \\
& x_{1}=x_{2}=\cdots=x_{h-1}=0, \quad x_{h}=1, \\
& 0 \leq x_{j} \leq 1, \quad h<j \leq N .
\end{aligned}
$$


and write

$$
\begin{aligned}
M & =\operatorname{conv}\left(P^{1} \cup P^{2} \cup \cdots P^{N}\right), \\
\bar{v} & =\min \left\{c^{T} x: x \in M\right\} .
\end{aligned}
$$

Note that $M$ is the projection to $\mathcal{R}^{N}$ of the feasible set for a system of $O\left(N^{2}\right)$ linear constraints in $O\left(N^{2}\right)$ variables. We have that $x \in M$ for any $0 / 1$ vector $x$ that satisfies (9) and therefore $\bar{v} \leq v^{Z}$.

Lemma $2.1 v^{Z}<2 \bar{v}$.

Proof. Let $\bar{x}$ be a solution to the linear program (13). It follows that there reals $\lambda_{h}$ such that

$$
0 \leq \lambda_{h}, \quad(1 \leq h \leq N), \quad \sum_{h=1}^{N} \lambda_{h}=1
$$

and, for each $1 \leq h \leq N$ with $\lambda_{h}>0$, a vector $x^{h} \in P^{h}$, such that

$$
\bar{x}=\sum_{h: \lambda_{h}>0} \lambda_{h} x^{h} .
$$

For $1 \leq h \leq N$ write

$$
v^{h}=\min \left\{c^{T} x: x \in P^{h}\right\}
$$

Suppose $\lambda_{h}>0$. It is straightforward to see that there is an optimal solution to (14) with at most one fractional variable. By rounding up this variable we obtain a feasible $0 / 1$ solution to the original knapsack problem. We therefore have by (8) and (10)

$$
v^{Z}-v^{h}<c_{h} \leq c^{T} x^{h}
$$

where the second inequality follows since $x_{h}^{h}=1$ again by (32). Consequently, writing

$$
\Lambda=\left\{1 \leq h \leq n: \lambda_{h}>0\right\},
$$

we have

$$
\begin{aligned}
v^{Z}-\bar{v} & =\sum_{h \in \Lambda} \lambda_{h} v^{Z}-\sum_{h \in \Lambda} \lambda_{h} c^{T} x^{h} \\
& \leq \sum_{h \in \Lambda} \lambda_{h} v^{Z}-\sum_{h \in \Lambda} \lambda_{h} v^{h} \\
& =\sum_{h \in \Lambda} \lambda_{h}\left(v^{Z}-v^{h}\right) \\
& <\sum_{h \in \Lambda} \lambda_{h} c^{T} x^{h}=\bar{v} .
\end{aligned}
$$




\subsection{Proof of Theorem 1.1.}

We begin with a technical result.

Lemma 2.2 Let $H \geq 1$ be an integer. suppose $S \subseteq\{1,2, \ldots, N\}$, and let $0 \leq \bar{x}_{j} \leq 1$ (j $j \in S$ ) be given values. Let $c^{\max }=\max _{j \in S}\left\{c_{j}\right\}, c^{\min }=\min _{j \in S}\left\{c_{j}\right\}$.

(a) Suppose first that

$$
\sum_{j \in S} \bar{x}_{j}=H
$$

Then there exist $0 / 1$ values $\hat{x}_{j}(j \in S)$ such that

$$
\begin{aligned}
\sum_{j \in S} a_{j} \hat{x}_{j} & \geq \sum_{j \in S} a_{j} \bar{x}_{j}, \quad \text { and } \\
\sum_{j \in S} c_{j} \hat{x}_{j} & \leq\left(1-\frac{1}{H}+\frac{c^{\max }}{H c^{\min }}\right) \sum_{j \in S} c_{j} \bar{x}_{j} .
\end{aligned}
$$

(b) Suppose next that

$$
\sum_{j \in S} \bar{x}_{j} \geq H
$$

Then there exist $0 / 1$ values $\hat{x}_{j}(j \in S)$ satisfying (20) and

$$
\sum_{j \in S} c_{j} \hat{x}_{j} \leq\left(1+\frac{c^{\max }}{H c^{\min }}\right) \sum_{j \in S} c_{j} \bar{x}_{j} .
$$

Proof. (a) Without loss of generality assume that $\bar{x}$ is an extreme point solution to the linear program

$$
\begin{array}{ll}
\min & \sum_{j \in S} c_{j} x_{j} \\
\text { s.t. } & \sum_{j \in S} a_{j} x_{j} \geq \sum_{j \in S} a_{j} \bar{x}_{j} \\
& \sum_{j \in S} x_{j}=H \\
& 0 \leq x_{j} \leq 1, \quad \forall j \in S .
\end{array}
$$

Consequently at most two of the values $\bar{x}_{j}, j \in S$, are fractional; but since $H$ is integral either zero or exactly two $\bar{x}_{j}$ are fractional. Thus, we can assume that there are indices $i, k \in S$ with

$$
\begin{aligned}
& 0<\bar{x}_{i}<1, \quad 0<\bar{x}_{k}<1, \quad \text { and } \\
& \bar{x}_{i}+\bar{x}_{k}=1 .
\end{aligned}
$$

Suppose (say) $a_{i} \geq a_{k}$. Then we can set $\hat{x}_{i}=1, \hat{x}_{k}=0$, and $\hat{x}_{j}=\bar{x}_{j}$ for all other $j$, thereby obtaining a $0 / 1$ vector $\hat{x}$ which satisfies (24) while increasing cost by at most

$$
c_{i}-c_{i}\left(\bar{x}_{i}\right)-c_{k}\left(\bar{x}_{k}\right)=\left(c_{i}-c_{k}\right)\left(1-\bar{x}_{i}\right) \leq c^{\max }-c^{\min } .
$$


Hence

$$
\frac{\sum_{j \in S} c_{j} \hat{x}_{j}-\sum_{j \in S} c_{j} \bar{x}_{j}}{\sum_{j \in S} c_{j} \bar{x}_{j}} \leq \frac{c^{\max }-c^{\text {min }}}{\sum_{j \in S} c_{j} \bar{x}_{j}} \leq \frac{c^{\max }-c^{\text {min }}}{H c^{\text {min }}}
$$

as desired.

(b) Proceeding in a way similar to (a) (using, instead of (25), $\sum_{j \in S} x_{j} \geq H$ ), it can be assumed that either zero, two or one of the $\bar{x}_{j}(j \in S)$ are fractional. If two are fractional the result is implied by (a). If there is only one fractional $\bar{x}_{j}$ then rounding up $\bar{x}$ provides a $0 / 1$ vector $\hat{x}$ that is feasible while increasing the cost by at most $c^{\max }$. Hence

$$
\frac{\sum_{j \in S} c_{j} \hat{x}_{j}-\sum_{j \in S} c_{j} \bar{x}_{j}}{\sum_{j \in S} c_{j} \bar{x}_{j}} \leq \frac{c^{\max }}{H c^{\min }},
$$

as desired.

Let $0<\epsilon<1$. Define $K$ as the smallest integer such that $(1+\epsilon)^{-K} \leq \epsilon$. Without loss of generality, $\epsilon$ is small enough that $K \approx \log (1 / \epsilon) / \epsilon$. Write $J=\lceil 1+1 / \epsilon\rceil$.

In what follows we still assume the ordering (8).

Definition 2.3 A signature will be a $K$-vector $\sigma$ such that $0 \leq \sigma_{i} \leq J$ for $i=1,2, \ldots, K$.

Let $1 \leq h \leq N$. For $k=1,2, \ldots, K$, let

$$
S^{h, k}=\left\{j: c_{h}(1+\epsilon)^{-(k-1)} \geq c_{j}>c_{h}(1+\epsilon)^{-k} \text { and } j>h\right\} .
$$

[Note: the "and" is redundant when $k>1$.] For each $1 \leq h \leq N$, and each signature $\sigma$, define

$$
\begin{gathered}
P^{h, \sigma}=\left\{x \in[0,1]^{N}: \quad \sum_{j=1}^{N} w_{j} x_{j} \geq b,\right. \\
x_{1}=x_{2}=\cdots=x_{h-1}=0, \quad x_{h}=1, \\
\sum_{j \in S^{h, k}} x_{j}=\sigma_{k}, \quad \forall k \text { such that } \sigma_{k}<J, \\
\sum_{j \in S^{h, k}} x_{j} \geq J, \quad \forall k \text { such that } \sigma_{k}=J \\
\} . \quad
\end{gathered}
$$

Lemma 2.4 For each $h$ and $\sigma$ there is a $0 / 1$ vector $\hat{x}^{h, \sigma}$ feasible for $\mathcal{K} \mathcal{M I N}$ such that $c^{T} \hat{x}^{h, \sigma} \leq$ $(1+\epsilon) \min \left\{c^{T} x: x \in P^{h, \sigma}\right\}$. As a result, $v^{Z} \leq(1+\epsilon) \min \left\{c^{T} x: x \in P^{h, \sigma}\right\}$.

Proof. Let $\bar{x} \in P^{h, \sigma}$. We $\hat{x}^{h, \sigma}$ as follows. First, for each $k$ such that $\sigma_{k}>0$, we obtain the values $\hat{x}_{j}^{h, \sigma}$ for each $j \in S^{h, k}$ by applying Lemma 2.2 with $S=S^{h, k}$. Here, note that when $\sigma_{k}<J$, the we have

$$
1-\frac{1}{\sigma_{k}}+\frac{c^{\max }}{\sigma_{k} c^{\min }} \leq \frac{c^{\max }}{c^{\min }} \leq 1+\epsilon
$$


by construction of the sets $S^{h, k}$. And if $\sigma_{k}=J$, we also have

$$
\left(1+\frac{c^{\max }}{J c^{\min }}\right) \leq 1+\epsilon
$$

by our choice for $J$. If on the other hand $\sigma_{k}=0$ we set $\hat{x}_{j}^{h, \sigma}=0$ for every $j \in S^{h, k}$.

Finally, define $T^{h}=\left\{j: c_{j} \leq(1+\epsilon)^{-K}\right\}$. The problem

$$
\begin{array}{ll}
\min & \sum_{j \in T^{h}} c_{j} x_{j} \\
\text { s.t. } & \sum_{j \in T^{h}} w_{j} x_{j} \geq \sum_{j \in T^{h}} w_{j} \bar{x}_{j} \\
& 0 \leq x_{j} \leq 1, \quad \forall j \in T^{h},
\end{array}
$$

is a knapsack problem, and hence it has an optimal solution $\tilde{x}$ with at most one fractional variable. We set $\hat{x}_{j}^{h, \sigma}=\left\lceil\tilde{x}_{j}\right\rceil$ for each $j \in T^{h}$; thereby increasing cost (from $\tilde{x}$ ) by less than $(1+\epsilon)^{-K} c_{h} \leq \epsilon c_{h}$ by definition of $K$.

In summary,

$$
\begin{aligned}
c^{T} \hat{x}^{h, \sigma}-c^{T} \bar{x} & =\sum_{k: \sigma_{k}>0}\left(\sum_{j \in S^{h, k}} c_{j} \hat{x}_{j}^{h, \sigma}-\sum_{j \in S^{h, k}} c_{j} \bar{x}_{j}\right)+\sum_{j \in T^{h}} c_{j}\left(\hat{x}_{j}^{h, \sigma}-\bar{x}_{j}\right) \\
& \leq \epsilon \sum_{k: \sigma_{k}>0} \sum_{j \in S^{h, k}} c_{j} \bar{x}_{j}+\epsilon c_{h} \\
& \leq \epsilon c^{T} \bar{x} .
\end{aligned}
$$

Here, (40) follows from Lemma 2.2, and by definition of the sets $S^{h, k}$, and (41) follows from the fact that $\bar{x}_{h}=1$, by definition of $P^{h, \sigma}$.

Consider the polyhedron

$$
Q=\operatorname{conv}\left(\bigcup_{h, \sigma} P^{h, \sigma}\right)
$$

Note that there are at most $(J+1)^{K} N=O\left((1 / \epsilon)^{O\left(1 / \epsilon^{2}\right)} N\right)$ polyhedra $P^{h, \sigma}$, and that each $P^{h, \sigma}$ is described by a system with $O(K+N)$ constraints in $N$ variables. Thus, $Q$ is the projection to $\mathcal{R}^{N}$ of the feasible set for a system with at most

$$
O\left(\left(\frac{1}{\epsilon}\right)^{O\left(1 / \epsilon^{2}\right)} N^{2}\right) \text { constraints in } O\left(\left(\frac{1}{\epsilon}\right)^{1 / \epsilon}\right) N^{2} \text { variables. }
$$

Furthermore, any $0 / 1$ vector $x$ that is feasible for the knapsack problem satisfies $x \in P^{h \sigma}$ for some $h$ and $\sigma$; in other words, $Q$ constitutes a valid relaxation to the knapsack problem.

Lemma $2.5 v^{Z} \leq(1+\epsilon) \min \left\{c^{T} x: x \in Q\right\}$. 
Proof. Let $\tilde{x} \in Q$. Then there exist reals $\lambda_{h, \sigma}$, for each $1 \leq h \leq N$ and $\sigma \subseteq\{1,2, \ldots, K\}$, such that

$$
0 \leq \lambda_{h, \sigma}, \forall h \text { and } \sigma, \text { and } \quad \sum_{h} \sum_{\sigma} \lambda_{h, \sigma}=1,
$$

and, for each $h$ and $\sigma$ with $\lambda_{h, \sigma}>0$, a vector $x^{h, \sigma} \in P^{h, \sigma}$, such that

$$
\tilde{x}=\sum_{h, \sigma: \lambda_{h, \sigma}>0} \lambda_{h, \sigma} x^{h, \sigma}
$$

Let $\Lambda=\left\{(h, \sigma): \lambda_{h, \sigma}>0\right\}$. Then

$$
\begin{aligned}
v^{Z}-c^{T} \tilde{x} & =\sum_{(h, \sigma) \in \Lambda} \lambda_{h, \sigma} v^{Z}-\sum_{(h, \sigma) \in \Lambda} \lambda_{h, \sigma} c^{T} x^{h, \sigma} \\
& =\sum_{(h, \sigma) \in \Lambda} \lambda_{h, \sigma}\left(v^{Z}-c^{T} x^{h, \sigma}\right) \\
& \leq \sum_{(h, \sigma) \in \Lambda} \lambda_{h, \sigma}\left(\epsilon c^{T} x^{h, \sigma}\right) \quad \text { (by Lemma 2.4) } \\
& =\epsilon c^{T} \tilde{x},
\end{aligned}
$$

as desired.

Note that the proofs above rely on term-by-term rounding of a solution to a linear program. As a consequence, we have the following result, whose proof we include for completeness, though it essentially follows from Lemmas 2.4 and 2.5 .

Corollary 2.6 For each $0<\epsilon<1$ there is an algorithm that computes a feasible $0 / 1$ solution to the minimum-knapsack problem, with value at most $(1+\epsilon) v^{Z}$. This algorithm requires the solution of $O\left((1 / \epsilon)^{O\left(1 / \epsilon^{2}\right)} N\right)$ linear programs with $O(N)$ variables and constraints.

Proof. By Lemma 2.4, for each $h$ and $\sigma$ there is a $0 / 1$ vector $\hat{x}^{h, \sigma}$ which is feasible for the knapsack problem, and such that $c^{T} \hat{x}^{h, \sigma} \leq(1+\epsilon) \min \left\{c^{T} x: x \in P^{h, \sigma}\right\}$. From among the set of all vectors $\hat{x}^{h, \sigma}$ select one of minimum cost; denoting this vector by $x^{Z}$ we have $c^{T} x^{Z} \leq$ $(1+\epsilon) \min \left\{c^{T} x: x \in P^{h, \sigma}\right\}$ for each $h$ and $\sigma$, and as a result $c^{T} x^{Z} \leq(1+\epsilon) \min \left\{c^{T} x: x \in Q\right\}$.

\section{Fixed-charge network flow problems}

In this section we consider the mixed-integer programs $\mathcal{F} \mathcal{X N}$ described in the introduction, in the case where the inner vertices $\mathcal{I}$ induce a path. In order to motivate the discussion we first discuss some special cases.

\subsection{Single inner node}

Let $\mathcal{I}=\{1\}$; we then have $\mathcal{A}=\delta^{+}(1) \cup \delta^{-}(1)$. To simplify notation we will index the arcs by the integers $1,2, \ldots, n=|\mathcal{A}|$. We assume that the arcs have been numbered so that

$$
f_{k}+c_{k} u_{k} \geq f_{k+1}+c_{k+1} u_{k+1}, \text { for } 1 \leq k<n .
$$


Problem $\mathcal{F} \mathcal{X N}$ can be written as

$$
\begin{aligned}
v^{Z}= & \min \sum_{j=1}^{n}\left(f_{j} y_{j}+c_{j} x_{j}\right), \\
& \sum_{j \in \delta^{+}(1)} x_{j}-\sum_{j \in \delta^{-}(1)} x_{j}=b_{1}, \\
& 0 \leq x_{j} \leq u_{j} y_{j} \quad \forall j, \\
& y_{j}=0 \text { or } 1, \quad \forall j .
\end{aligned}
$$

Given a vector $(x, y)$ feasible for $\mathcal{F} \mathcal{X N}$ an arc $j$ is called slack if $y_{j}=1$ and $0<x_{j}<u_{j} y_{j}$. Otherwise the arc is called tight. The following result is routine.

Lemma 3.1 In any extreme point solution to (48)-(51) there is at most one slack arc.

\subsubsection{Achieving a bound of 2}

For each pair of indices $1 \leq h, i \leq n$ (including $i=h$ ), consider the polyhedron $D^{h, i}$ defined by

$$
\begin{gathered}
\sum_{j \in \delta^{+}(1)} x_{j}-\sum_{j \in \delta^{-}(1)} x_{j}=b_{1} ; \quad 0 \leq y_{j} \leq 1 \forall j \in \mathcal{A}, \\
y_{i}=y_{h}=1 ; \quad \text { if } h \neq i \text { then } y_{g}=0 \forall g \neq i \text { with } g<h ; \\
\quad \text { if } h=i \text { then } y_{g}=0 \forall g \neq h, \\
0 \leq x_{i} \leq u_{i} y_{i} ; \quad x_{g}=u_{g} y_{g} \forall g \neq i .
\end{gathered}
$$

Now as a consequence of Lemma 3.2 we have:

Lemma 3.2 Let $(\hat{x}, \hat{y}) \neq(0,0)$ be an extreme point solution to (48)-(51). Then there exist indices $1 \leq h, i \leq n$ such that $(\hat{x}, \hat{y}) \in D^{h, i}$.

Proof. We have $\hat{y} \neq 0$. If there is a unique index $j$ with $\hat{y}_{j}=1$, then set $i=h=j$ and we are done. Otherwise, (using Lemma 3.1) let $h$ be the minimum index $j$ with $\hat{y}_{j}=1$ and $\hat{x}_{j}=u_{j} y_{j}$. Again using Lemma 3.1 there is at most one index $j$ with $\hat{y}_{j}=1$ and $0<\hat{x}_{j}<u_{j}$; we set $i$ equal to that index if it exists, and we set $i=h$ otherwise.

Define:

$$
Q= \begin{cases}\operatorname{conv}\left(\bigcup_{h, i} D^{h, i}\right) & \text { if } b_{1} \neq 0 \\ \operatorname{conv}\left((0,0) \cup \bigcup_{h, i} D^{h, i}\right) & \text { otherwise. }\end{cases}
$$

As a consequence of Lemma 3.1 we have that if $(\hat{x}, \hat{y})$ is feasible for $(48)-(51)$ then $(\hat{x}, \hat{y}) \in Q$.

Lemma 3.3 For each pair of indices $1 \leq h, i \leq n$,

$$
v^{Z} \leq 2 \min \left\{c^{T} x+f^{T} y:(x, y) \in D^{h, i}\right\} .
$$


Proof. Let $(\bar{x}, \bar{y})$ be an optimal extreme point solution to $\min \left\{c^{T} x+f^{T} y:(x, y) \in D^{h, i}\right\}$, and assume by contradiction that there exist indices $p \neq q$ with $0<\bar{y}_{p}<1$ and $0<\bar{y}_{q}<1$. Then $p, q \neq h$ and $p, q \neq i$. Consequently, $\bar{x}_{p}=u_{p} \bar{y}_{p}$ and $\bar{x}_{q}=u_{q} \bar{y}_{q}$, and therefore $\bar{x}_{p}>0$ and $\bar{x}_{q}>0$.

Assume first that $p \in \delta^{+}(1)$ and $q \in \delta^{+}(1)$. Then for real $\delta$ small enough in absolute value, we can reset

$$
\begin{array}{ll}
y_{p} \leftarrow \bar{y}_{p}+\delta, & x_{p} \leftarrow \bar{x}_{p}+u_{p} \delta, \\
y_{q} \leftarrow \bar{y}_{q}-\frac{u_{p}}{u_{q}} \delta, & x_{q} \leftarrow \bar{x}_{q}-u_{p} \delta,
\end{array}
$$

maintaining feasibility for $D^{h, i}$, contradicting the assumption that $(\bar{x}, \bar{y})$ is an extreme point. The remaining cases (both $p$ and $q$ in $\delta^{-}(1)$, one each in $\delta^{+}(1)$ and $\delta^{-}(1)$ ) are similarly handled.

Thus, there is a unique index $i$ with $0<\bar{y}_{g}<1$. Setting $y_{g}=1$ (and keeping all other variables unchanged) yields a feasible solution to $\mathcal{F} \mathcal{X N}$; since $g>h$ and all costs are nonnegative this change at most doubles the cost.

As a corollary we now have $v^{Z} \leq 2 \min \left\{c^{T} x+f^{T} y:(x, y) \in Q\right\}$.

\subsubsection{Achieving a bound of $1+\epsilon$}

Let $0<\epsilon<1$. In order to improve the IP/LP ratio from 2 to $1+\epsilon$, we apply the technique used in Section 2.1 to prove the corresponding result for the minimum knapsack problem.

In the context of the single inner-node problem, this works out as follows: rather than constructing a disjunction based on the polyhedra $D^{h, i}$ as defined above, instead we construct a disjunction using polyhedra $D^{h, i, \sigma}$, where $\sigma$ is a signature (see Definition 2.3). The polyhedra $D^{h, i, \sigma}$ are obtained by adapting the definition of $D^{h, i}$ given above, in particular, equation (54), in order to mirror equations (33), (34). The proof that the resulting disjunction proves the desired $1+\epsilon$ bound is much like the proof of Lemmas 2.4, 3.3.

\subsection{Two inner nodes}

We assume $\mathcal{I}$ consists of nodes 1 and 2 , and that the arc between 1 and 2 is $(1,2)$. For $i=1,2$, write $\Delta(i)=\delta^{-}(i) \cup \delta^{+}(i) \backslash(1,2)$. The following result is straightforward.

Lemma 3.4 In an optimal solution to $(x, y)$ for $\mathcal{F} \mathcal{X N}$, without loss of generality either

(a) $y_{12}=1$ and $x_{12}=u_{12}$, or $y_{12}=0$ and $x_{12}=0$; and in either case there $i s$ at most one slack arc in $\Delta(1)$ and at most one slack arc in $\Delta(2)$.

(b) $y_{1}=1$ and $0<x_{1}<u_{1}$; and there is at most one slack arc in $\Delta(1) \cup \Delta(2)$.

We now show how to generate a polynomial-size relaxation that yields an IP/LP ratio of at most 2. Using Lemma 3.4 it is straightforward to do so with a disjunction with $O\left(n^{4}\right)$ terms relying on the results of the previous section. However, we will show how achieve the same bound with $O\left(n^{2}\right)$ terms using a system of nested disjunctions.

We number the arcs in $\Delta(1) \cup \Delta(2)$ as $1,2, \ldots, n-1$, where the indices reflect non-increasing values of $f_{j}+c_{j} u_{j}$ (we will still refer to the arc between 1 and 2 as $(1,2)$, however). 
Let $h, i \in \Delta(1)$. For $\phi=0$ or $\phi=1$, consider the polyhedron $D_{1, \phi}^{h, i} \subseteq \mathcal{R}_{+}^{\Delta(1)} \times \mathcal{R}_{+}^{\Delta(1)} \times \mathcal{R}_{+}$defined by:

$$
\begin{aligned}
& \sum_{j \in \delta^{+}(1) \backslash(1,2)} x_{j}-\sum_{j \in \delta^{-}(1)} x_{j}-\left(b_{1}-u_{12} \phi\right) \alpha=0, \quad 0 \leq y_{j} \leq \alpha, \quad \forall j \in \Delta(1) \\
& y_{h}=y_{i}=\alpha ; \text { if } h \neq i \text { then } y_{g}=0 \quad \forall g \in \Delta(1) \text { with } g \neq i \text { and } g<h \text {; } \\
& \text { if } h=i \text { then } y_{g}=0 \forall g \in \Delta(1) \backslash h \text {, } \\
& 0 \leq x_{i} \leq u_{i} y_{i} ; \quad x_{g}=u_{g} y_{g} \quad \forall g \neq i \text { with } g \in \Delta(1) \text {, } \\
& 0 \leq \alpha \text {. }
\end{aligned}
$$

Note: The quantity $\alpha$ is a homogenization factor, the user should think of it as taking values either 1 or 0 (in the latter case all $x$ and $y$ will take value zero). Also, when $\phi=1$ (resp., 0) (58) corresponds to the flow conservation constraint at node 1 assuming the arc $(1,2)$ carries flow $u_{12}$ (resp., 0), both after homogenization.

Similarly, for each pair $h, i \in \Delta(2)$, and $\phi=0$ or $\phi=1$, consider the polyhedron $D_{2, \phi}^{h, i} \subseteq$ $\mathcal{R}_{+}^{\Delta(2)} \times \mathcal{R}_{+}^{\Delta(2)} \times \mathcal{R}_{+}$defined by:

$$
\begin{aligned}
& \sum_{j \in \delta^{+}(2)} x_{j}-\sum_{j \in \delta^{-}(2) \backslash(1,2)} x_{j}-\left(b_{2}+u_{12} \phi\right) \beta=0, \quad 0 \leq y_{j} \leq \beta, \quad \forall j \in \Delta(2) \\
& \begin{array}{l}
y_{h}=y_{i}=\beta ; \quad \text { if } h \neq i \text { then } y_{g}=0 \quad \forall g \in \Delta(2) \text { with } g \neq i \text { and } g<h ; \\
\quad \text { if } h=i \text { then } y_{g}=0 \forall g \in \Delta(2) \backslash h, \\
0 \leq x_{i} \leq u_{i} y_{i} ; \quad x_{g}=u_{g} y_{g} \quad \forall g \neq i \quad \text { with } g \in \Delta(2), \\
0 \leq \beta .
\end{array}
\end{aligned}
$$

Finally, writing $\Delta=\Delta(1) \cup \Delta(2)$, then for each pair of indices $h, i \in \Delta$ consider the polyhedron $T^{h, i}$ defined by:

$$
\begin{aligned}
& \sum_{j \in \delta^{+}(1) \backslash(1,2)} x_{j}-\sum_{j \in \delta^{-}(1)} x_{j}-b_{1} \gamma=0, \sum_{j \in \delta^{+}(2)} x_{j}-\sum_{j \in \delta^{-}(2) \backslash(1,2)} x_{j}-b_{2} \gamma=0, \\
& 0 \leq y_{j} \leq \gamma \forall j \in \Delta, \\
& y_{12}=\gamma, \quad 0 \leq x_{12} \leq u_{12} y_{12}, \\
& y_{h}=y_{i}=\gamma ; \quad \text { if } h \neq i \text { then } y_{g}=0 \forall g \in \Delta \text { with } g \neq i \text { and } g<h ; \\
& \quad \text { if } h=i \text { then } y_{g}=0 \forall g \in \Delta \backslash h, \\
& 0 \leq x_{i} \leq u_{i} y_{i} ; \quad x_{g}=u_{g} y_{g} \forall g \neq i .
\end{aligned}
$$

Roughly speaking the $D_{k, \delta}^{h, i}$ polyhedra capture case (a) of Lemma 3.4 whereas the $T^{h, i}$ will be shown to capture case (b).

Our overall formulation can now be given. For $k=1,2$ we will use the notation $(x(k), y(k))$ to denote an $(x, y)$ vector with entries for arcs in $\Delta(k)$. For convenience of notation, we will also assume that $b_{1} \neq 0$ and $b_{2} \neq 0$; we will indicate later how to handle the other cases as well. Using (somewhat abused) vector notation, the formulation is: 


$$
\begin{aligned}
& \left(x(1), y(1), x_{12}, y_{12}, x(2), y(2), 1\right)= \\
& \sum_{\phi=0,1} \sum_{i, h \in \Delta(1)}\left(x(1)^{i, h, \phi}, y(1)^{i, h, \phi}, u_{12} \phi \alpha^{i, h, \phi}, \phi \alpha^{i, h, \phi}, x(2), y(2), \alpha^{i, h, \phi}\right)+ \\
& \sum_{i, h \in \Delta}\left(\hat{x}(1)^{i, h}, \hat{y}(1)^{i, h}, \hat{x}_{12}^{i, h}, \hat{y}_{12}^{i, h}, \hat{x}(2)^{i, h}, \hat{y}(2)^{i, h}, \gamma^{i, h}\right) \\
& \left(x(1)^{i, h, \phi}, y(1)^{i, h, \phi}, \alpha^{i, h, \phi}\right) \in D_{1, \phi}^{h, i}, \quad \forall \phi, \text { and } \forall i, h \in \Delta(1) \\
& \left(\hat{x}(1)^{i, h}, \hat{y}(1)^{i, h}, \hat{x}_{12}^{i, h}, \hat{y}_{12}^{i, h}, \hat{x}(2)^{i, h}, \hat{y}(2)^{i, h}, \gamma^{i, h}\right) \in T^{h, i}, \quad \forall i, h \in \Delta \\
& \left(x(2), y(2), \alpha^{i, h, \phi}\right)=\sum_{\phi^{\prime}=0,1} \sum_{i^{\prime}, h^{\prime} \in \Delta(2)}\left(x(2)^{i^{\prime}, h^{\prime}, \phi^{\prime}}, y(2)^{i^{\prime}, h^{\prime}, \phi^{\prime}}, \beta^{i^{\prime}, h^{\prime}, \phi^{\prime}}\right) \\
& \text { for all } \phi=0,1 \text {, and } i, h \in \Delta(1) \\
& \left(x(2)^{i^{\prime}, h^{\prime}, \phi^{\prime}}, y(2)^{i^{\prime}, h^{\prime}, \phi^{\prime}}, \beta^{i^{\prime}, h^{\prime}, \phi^{\prime}}\right) \in D_{2, \phi^{\prime}}^{h^{\prime}, i^{\prime}}, \quad \forall \phi^{\prime}=0,1 \text {, and } i^{\prime}, h^{\prime} \in \Delta(2) .
\end{aligned}
$$

Note that the vector equation (70) includes the condition $\sum_{\phi=0,1} \sum_{i, h \in \Delta(1)} \alpha^{i, h, \phi}+\sum_{i, h \in \Delta} \gamma^{i, h}=1$. Thus, (70) describes a disjunction. The second term in the right-hand side of (70) corresponds to case (b) of Lemma 3.4, whereas the first term corresponds to case (a) and we disjunct on the arcs of $\Delta(1)$ and whether $y_{12}=0$ or 1 .

Moreover, for a given $\phi=0,1$, and $i, h \in \Delta(1)$, (73) requires that

$$
\alpha^{i, h, \phi}=\sum_{\phi^{\prime}=0,1} \sum_{i^{\prime}, h^{\prime} \in \Delta(2)} \beta^{i^{\prime}, h^{\prime}, \phi^{\prime}}
$$

Thus (73) is also a disjunction (more precisely: it is a disjunction when $\alpha^{i, h, \phi}>0$, after scaling the equation by $\left.1 / \alpha^{i, h, \phi}\right)$.

Based on these observations it follows that (70)-(74) describes a valid relaxation for $\mathcal{F} \mathcal{X N}$. We can now present the rounding result.

Lemma $3.5 v^{Z} \leq 2 \min \left\{c^{T} x+f^{T} y:(x, y)\right.$ satisfies (70)-(74) $\}$.

Proof. Consider an extreme point optimal solution $\left(x(1), y(1), x_{12}, y_{12}, x(2), y(2)\right)$ to the relaxation with constraints (70)-(74) chosen in addition so that the sum of $\alpha^{i, h, \phi}$ is maximum.

Note first that for each $\phi^{\prime}=0,1$, and $i^{\prime}, h^{\prime} \in \Delta(2)$ we can "round" $\left(x(2)^{i^{\prime}, h^{\prime}, \phi^{\prime}}, y(2)^{i^{\prime}, h^{\prime}, \phi^{\prime}}, \beta^{i^{\prime}, h^{\prime}, \phi^{\prime}}\right)$ to a vector feasible for $D_{2, \phi^{\prime}}^{h^{\prime}, i^{\prime}}$ such that $y_{u, v}=0$ or $\beta^{i^{\prime}, h^{\prime}, \phi^{\prime}}$ for every $(u, v) \in \Delta(2)$, while at most doubling the cost. This is analogous to the proof of Lemma 3.3 in the single inner-node case.

A similar observation applies for each $\phi=0,1$, and $i, h \in \Delta(1)$ - the vector $\left(x(1)^{i, h, \phi}, y(1)^{i, h, \phi}, \alpha^{i, h, \phi}\right)$ can be rounded to a vector feasible for $D_{1, \phi}^{h, i}$ such that $y_{u, v}=0$ or $\alpha^{i, h, \phi}$ for every $(u, v) \in \Delta(1)$, while at most doubling the cost. 
Thus, by (70), the proof of the Lemma will be complete if we can prove a similar rounding result for each vector $\left(\hat{x}(1)^{i, h}, \hat{y}(1)^{i, h}, \hat{x}_{12}^{i, h}, \hat{y}_{12}^{i, h}, \hat{x}(2)^{i, h}, \hat{y}(2)^{i, h}, \gamma^{i, h}\right)$ where $i, h \in \Delta$, and $\gamma^{i, h}>0$.

Following the proof of Lemma 3.3, this result would follow if we can show that there is at most one $\operatorname{arc}(u, v) \in \Delta$ such that $0<\hat{y}_{u v}<\gamma^{i, h}$. In turn, this is clear if $0<\hat{x}_{12}<\gamma^{i, h} u_{12}$. So assume not. Then either $0=\hat{x}_{12}$, or $\hat{x}_{12}=\gamma^{i, h} u_{12}$. In the first case by nonnegativity of the costs we can assume without loss of generality that $0=\hat{y}_{12}$.

But then in either case term (72) in the disjunction corresponding to $(i, h)$ corresponds to an instance of case (a) (with $\phi=0$ and $\phi=1$, respectively). It follows that there is an equivalent representation of $\left(x(1), y(1), x_{12}, y_{12}, x(2), y(2)\right)$ where the sum of $\alpha^{i, h, \phi}$ has been increased by $\gamma^{i, h}$, a contradiction.

Above we had assumed that $b_{1} \neq 0$ and that $b_{2} \neq 0$. If not, in order to make the construction complete we simply need to add terms to the disjunctions given above corresponding to solutions where all arcs in $\Delta(1)$ or $\Delta(2)$ are set at zero (zero flow and zero $y$ variables). It is straightforward to show that Lemma 3.5 still holds, as the proof given above proceeded on a term-by-term basis.

\subsection{General case}

We only sketch the construction in the case that $\mathcal{I}$ induces a general path, which we denote by $\mathcal{P}$.

In this case one can obtain a result similar to Lemma 3.4. An extreme point solution $(\hat{x}, \hat{y})$ to $\mathcal{F} \mathcal{X N}$ (where by definition $\hat{y}$ is a $0 / 1$-vector) can be decomposed into intervals: we have $\mathcal{P}=\mathcal{P}_{1} \cup \mathcal{P}_{2} \cup \ldots \mathcal{P}_{k}$, where

1. The $\mathcal{P}_{i}$ are vertex-disjoint supbaths of $\mathcal{P}$.

2. For any arc $j \in \mathcal{P}, 0<\hat{x}_{j}<u_{j}$ if and only if both ends of $j$ belong to the same subpath $\mathcal{P}_{i}$.

The formulation of the relaxation is similar to that given by (70)-(74), with the difference that there are $|\mathcal{I}|-1$ nested levels of disjunctions. The building blocks of the disjunctions correspond to intervals; for each interval we need to specify indices $i, h$ as above, as well as up to two values $\phi, \phi^{\prime}$, each corresponding to the condition (flow at zero or at upper bound) of each arc connecting the interval to the rest of $\mathcal{P}$.

As was the case for the minimum-knapsack problem, the constructions can be used to yield approximation algorithms:

Corollary 3.6 Given an instance of $\mathcal{F} \mathcal{X N}$ where the inner vertices induce a path, for each $0<$ $\epsilon<1$ there is an algorithm that computes a feasible $0 / 1$ of cost at most $(1+\epsilon) v^{Z}$. This algorithm solves $O\left((1 / \epsilon)^{O\left(1 / \epsilon^{2}\right)}(1+|\mathcal{O}|)^{2}|\mathcal{I}|^{2}\right)$ linear programs in $O(|\mathcal{O}|+|\mathcal{I}|)$ variables.

There are several ways in which Theorem 1.2 and Corollary 3.6 can be generalized, always relying on the basic disjunctive technique given above. An open case is that where the inner nodes induce a graph of fixed tree-width (the fixed path-width case is an easy extension). See [15]. 


\section{Maximum knapsack}

In this section we will present the results on the maximum knapsack problem. First we will show that using valid inequalities with "small" coefficients then the LP/IP ratio can remain arbitrarily close to 2 . Then we we will discuss our use of disjunctions, and finally we will provide our analysis of clique inequalities in the case that the coefficients $w_{j}$ are not "large".

\subsection{Valid inequalities with small coefficients}

Consider the knapsack instance with $N=n+1$ where

$$
\begin{aligned}
& p_{1}=p_{2}=\ldots=p_{n}=1, p_{n+1}=n, \\
& w_{1}=w_{2}=\ldots=w_{n}=1, w_{n+1}=n^{2}-\lfloor\sqrt{n}\rfloor, \\
& b=n^{2} .
\end{aligned}
$$

Write $s=\lfloor\sqrt{n}\rfloor$. Clearly,

$$
v^{Z}=n+s \sim n
$$

The lifted cover inequality,

$$
\sum_{j=1}^{n} x_{j}+(n-s) x_{n+1} \leq n
$$

defines a facet of $\operatorname{conv}(\mathcal{K} \mathcal{M} \mathcal{A} \mathcal{X})$. This suggests that the set of valid inequalities with 'small' coefficients may not adequately approximate $\operatorname{conv}(\mathcal{K} \mathcal{M} \mathcal{A} \mathcal{X})$, but it is not clear how large the shortfall is. Consider the valid inequalities with left-hand side coefficients 0 or 1 , which are as follows:

$$
\begin{array}{ll}
\forall A \subseteq\{1,2, \ldots, n\}, & \sum_{j \in A} x_{j} \leq|A|, \text { and } \\
\forall A \subseteq\{1,2, \ldots, n\}, & \sum_{j \in A} x_{j}+x_{n+1} \leq \begin{cases}|A|+1, & \text { if }|A| \leq s, \\
|A|, & \text { otherwise. }\end{cases}
\end{array}
$$

Let $x^{*}$ be the point defined by $x_{j}^{*}=1-1 / s$, for $1 \leq j \leq n+1$. It is not difficult to show that $x^{*}$ satisfies all inequalities (76) as well as (6), for $n$ large enough. At the same time, we have $\sum_{j=1}^{n+1} p_{j} x_{j}^{*}=n-s+n-n / s \sim 2 n$. However, a stronger result can be proved.

Lemma 4.1 Let $0<\epsilon<1$. Consider the knapsack instance with $N=n+1$ where

$$
\begin{aligned}
& p_{1}=p_{2}=\ldots=p_{n}=1, p_{n+1}=n \\
& w_{1}=w_{2}=\ldots=w_{n}=1, w_{n+1}=n^{2}-n / 2, \\
& b=n^{2}
\end{aligned}
$$

Let

$$
\sum_{j=1}^{n+1} \alpha_{j} x_{j} \leq \beta
$$

be a valid inequality for the knapsack polytope defined by (77)-(79), where $\alpha_{j} \in\left\{0,1, \ldots,\left\lfloor n^{1-\epsilon}\right\rfloor\right\}$ for $1 \leq j \leq n+1$. Consider the point $\hat{x}$ with $\hat{x}_{j}=1-2 n^{-\epsilon}$ for $1 \leq j \leq n+1$. Then for $n$ large enough, $\hat{x}$ is feasible for the continuous relaxation of $\mathcal{K} \mathcal{M} \mathcal{A} \mathcal{X}$ and satisfies (80). 
Proof. The first assertion is clear. To prove the second, let $B$ denote the sum of the $n / 2$ largest $\alpha_{j}$ chosen among the indices $1 \leq j \leq n$. Then, without loss of generality,

$$
\beta=\max \left\{\sum_{j=1}^{n} \alpha_{j}, B+\alpha_{n+1}\right\} .
$$

Consider first the case where there are at least $n / 2$ positive $\alpha_{j}$ with $1 \leq j \leq n$. Then

$$
\begin{aligned}
& \sum_{j=1}^{n+1} \alpha_{j} \hat{x}_{j}< \\
& \sum_{j=1}^{n} \alpha_{j}\left(1-2 n^{-\epsilon}\right)+n^{1-\epsilon} \leq \sum_{j=1}^{n} \alpha_{j}-2 n^{-\epsilon}\left(\frac{n}{2}\right)+n^{1-\epsilon}=\sum_{j=1}^{n} \alpha_{j},
\end{aligned}
$$

as desired. Suppose there are fewer than $n / 2$ positive $\alpha_{j}$ with $1 \leq j \leq n$. Then $\sum_{j=1}^{n+1} \alpha_{j} \hat{x}_{j}$ is less than $B+\alpha_{n+1}$.

\subsection{Using disjunctions}

Let $r=\frac{\sqrt{19}-2}{3}$. In this section we describe a simple disjunction which is guaranteed to result in an LP value at most $(1+r) v^{Z} \approx 1.79 v^{Z}$. Without loss of generality, assume that the optimal solution to the continuous relaxation of $\mathcal{K} \mathcal{M} \mathcal{A} \mathcal{X}$ has value 2 . Thus, $v^{Z} \geq 1$.

Assume that $2>(1+r) v^{Z}$. Since $w_{j} \leq b$ for each $j$, we have that $p_{j}<2 /(1+r)$ for each $j$. Define

$$
\Omega=\left\{j: p_{j} \geq r\right\}, \text { and } \tilde{w}=\min \left\{w_{j}: j \in \Omega\right\} .
$$

Let $j^{*} \in \Omega$ be such that $w_{j^{*}}=\tilde{w}$ (if $\Omega=\emptyset j^{*}$ will be irrelevant). We have that

$$
\mathcal{K} \mathcal{M} \mathcal{A X} \subseteq \operatorname{conv}\left(\mathcal{L}^{2} \cup \mathcal{L}^{1} \cup \mathcal{L}^{0}\right)
$$

where $\mathcal{L}^{i}, 0 \leq i \leq 2$ are the following convex polyhedra. First, $\mathcal{L}^{2}$ is the set of solutions to the system:

$$
\begin{aligned}
& \sum_{j=1}^{N} w_{j} x_{j} \leq b, \\
& \sum_{j \in \Omega} x_{j} \geq 2, \\
& 0 \leq x_{j} \leq 1, \quad \forall j .
\end{aligned}
$$

Similarly, $\mathcal{L}^{1}$ is the set of solutions to the system:

$$
\begin{aligned}
& \sum_{j=1}^{N} w_{j} x_{j} \leq b \\
& \sum_{j \in \Omega} x_{j}=1 \\
& x_{j}=0, \quad \forall j \neq j^{*} \text { with } w_{j}+\tilde{w}>b, \\
& 0 \leq x_{j} \leq 1, \quad \forall j .
\end{aligned}
$$


Finally, $\mathcal{L}^{0}$ is the set of solutions to the system:

$$
\begin{aligned}
& \sum_{j=1}^{N} w_{j} x_{j} \leq b, \\
& x_{j}=0 \quad \forall j \in \Omega, \\
& 0 \leq x_{j} \leq 1, \quad \forall j .
\end{aligned}
$$

It is clear that (83) holds. Further, we can separate from $\operatorname{conv}\left(\mathcal{L}^{2} \cup \mathcal{L}^{1} \cup \mathcal{L}^{0}\right)$ in polynomial time. Now, if $\mathcal{L}^{2} \neq \emptyset$, there exist distinct $i(1), i(2) \in \Omega$ with $w_{i(1)}+w_{i(2)} \leq b$ (e.g. the two indices in $\Omega$ with smallest $w_{j}$ ). In that case, $v^{Z} \geq 2 r$, and so the LP to IP ratio is at most

$$
\frac{2}{2 r} \leq 1+r
$$

In what follows we will assume $\mathcal{L}^{2}=\emptyset$, and show that

$$
\max \left\{\sum_{j} p_{j} x_{j}: x \in \mathcal{L}^{k}\right\} \leq(1+r) v^{Z} \text { for } k=0,1
$$

as desired.

Consider first $k=1$. Let $\hat{x}$ be an optimal solution to $\max \left\{\sum_{j} p_{j} x_{j}: x \in \mathcal{L}^{1}\right\}$, and suppose that $\sum_{j} p_{j} \hat{x}_{j}>1+r$. Since

$$
\sum_{j \in \Omega} p_{j} \hat{x}_{j} \leq \max _{j \in \Omega} p_{j}
$$

(by (88)), and

$$
\sum_{j \in \Omega} w_{j} \hat{x}_{j} \geq \tilde{w}
$$

(by (88) and the definition of $\tilde{w}$ ), we have

$$
\begin{aligned}
& \sum_{j \notin \Omega} p_{j} \hat{x}_{j} \geq 1+r-\frac{2}{1+r}, \text { and } \\
& \sum_{j \notin \Omega} w_{j} \hat{x}_{j} \leq b-\tilde{w} .
\end{aligned}
$$

Hence there is a set $S$ disjoint from $\Omega$ with

$$
\begin{aligned}
& \sum_{j \in S} p_{j} \geq \frac{1}{2}\left(1+r-\frac{2}{1+r}\right), \text { and } \\
& \sum_{j \in S} w_{j} \leq b-\tilde{w} .
\end{aligned}
$$

Therefore, setting $x_{j}=1$ if $j \in S \cup\left\{j^{*}\right\}$, and $x_{j}=0$ otherwise, yields a feasible solution to the knapsack problem with value at least

$$
\frac{1}{2}\left(1+r-\frac{2}{1+r}\right)+r \geq \frac{2}{1+r}
$$


as a simple calculation shows, as desired.

Next we consider $\mathcal{L}^{0}$. Clearly, $\max \left\{\sum_{j} p_{j} x_{j}: x \in \mathcal{L}^{0}\right\}$ is simply the continuous relaxation of a knapsack problem. As is well known, there is an optimal solution $\tilde{x}$ to this problem with the following structure: for some set $S, \tilde{x}_{j}=1$ for all $j \in S ; 0<x_{k}<1$ for at most one additional index $k$ (note that $k \notin \Omega$, by (93)), and $x_{j}=0$ otherwise. Hence, the value of the relaxation is strictly less than

$$
v^{Z}+\max _{j \notin \Omega}\left\{p_{j}\right\}<v^{Z}+r \leq(1+r) v^{Z},
$$

as desired.

\subsection{Knapsacks with small coefficients}

In this section we prove Theorem 1.3. Let $0 \leq \lambda<1$ be given. Consider the linear programming relaxation of $\mathcal{K} \mathcal{M} \mathcal{A} \mathcal{X}$

$$
\begin{aligned}
v^{*}= & \max \sum_{j=1}^{N} p_{j} x_{j}, \\
\text { s.t. } & \sum_{j=1}^{N} w_{j} x_{j} \leq b, \\
& x \in[0,1]^{N} .
\end{aligned}
$$

We can obtain an optimal solution $x^{*}$ to this linear program as follows. Assume without loss of generality that that $p_{1} / w_{1} \geq p_{2} / w_{2} \geq \ldots p_{N} / w_{N}$. Then, for some integer $n \geq 1$, we have

$$
\begin{aligned}
& x_{j}^{*}=1, \text { for } 1 \leq j \leq n, \\
& x_{n+1}^{*}=\frac{b-\sum_{j=1}^{n} w_{j}}{w_{n+1}}, \\
& x_{j}^{*}=0, \text { for } n+1<j \leq N .
\end{aligned}
$$

If $x_{n+1}^{*}=0$ or 1 then $v^{Z}=v^{*}$ and there is nothing left to prove. In what follows we assume $0<x_{n+1}^{*}<1$.

Without loss of generality we assume $\lambda>1 / 2$. Write $\kappa=2 \lambda-1$. Note that $0<\kappa<1$. We will choose

$$
\epsilon=\min \left\{\frac{1-\kappa}{4(1+\kappa)}, \frac{1}{138}\right\} \text {. }
$$

Note that $\epsilon<1-\kappa$.

Our proof of Theorem 1.3 will proceed in a number of steps. We will assume by contradiction that $v^{\omega}>(2-\epsilon) v^{Z}$. Without loss of generality, we will assume that the $p_{j}$ have been scaled so that $v^{*}=2$ (and thus $v^{Z}>1$ ). Likewise, we will assume the $w_{j}$ have been scaled so that $b=2$. We next prove some structural results (Lemma 4.2 through Lemma 4.5) that follow from these assumptions.

Lemma 4.2 (a) $\max \left\{\sum_{j=1}^{n} p_{j}, \max _{k}\left\{p_{k}\right\}\right\}<1+\epsilon$. (b) $\min \left\{\sum_{j=1}^{n} p_{j}, p_{n+1}\right\}>1-\epsilon$. (c) $x_{n+1}^{*}>1-2 \epsilon$. 
Proof. (a) Assume that $p_{k} \geq 1+\epsilon$ for some $k$. The solution with $x_{k}=1$ and $x_{j}=0$ for all other $j$ is feasible, and thus

$$
\frac{v^{*}}{v^{Z}} \leq \frac{2}{1+\epsilon} \leq 2-\epsilon
$$

(since $0 \leq \epsilon \leq 1$ ) a contradiction. Similarly, $\sum_{j=1}^{n} p_{j}<1+\epsilon$. To prove (b), note that

$$
2=\sum_{j=1}^{n} p_{j}+x_{n+1}^{*} p_{n+1}<\sum_{j=1}^{n} p_{j}+1+\epsilon
$$

yielding the bound on $\sum_{j=1}^{n} p_{j}$. The bound on $p_{n+1}$ is similarly obtained from the first equation in (106). This equation also yields

$$
x_{n+1}^{*}=\frac{2-\sum_{j=1}^{n} p_{j}}{p_{n+1}}>\frac{1-\epsilon}{1+\epsilon}>1-2 \epsilon,
$$

thereby proving (c).

Write $\Delta=w_{n+1}-x_{n+1}^{*} w_{n+1}$. By definition of $\kappa, \lambda b=2 \lambda=1+\kappa$, so by Lemma $4.2(\mathrm{c}), \Delta<$ $2 \epsilon w_{n+1} \leq 2 \epsilon \lambda b=2 \epsilon(1+\kappa)$. Also, note that $2=\sum_{j=1}^{n} w_{j}+x_{n+1}^{*} w_{n+1}$, so

$$
\sum_{j=1}^{n} w_{j}-\Delta=b-x_{n+1}^{*} w_{n+1}-\Delta=b-w_{n+1} \geq 0
$$

Define

$$
j^{*}=\min \left\{i: \sum_{j=1}^{i} w_{j} \geq \sum_{j=1}^{n} w_{j}-\Delta\right\} .
$$

Lemma 4.3 (a) $\sum_{j=1}^{j^{*}-1} p_{j}<2 \epsilon$. (b) $\sum_{j=j^{*}+1}^{n} p_{j}<2 \epsilon$.

Proof. (a) The vector $x$ with $x_{1}=\ldots=x_{j^{*}-1}=1, x_{n+1}=1$, and $x_{j}=0$ for all other $j$, is feasible, by definition of $j^{*}$, which implies $\sum_{j=1}^{j^{*}-1} p_{j}+p_{n+1}<1+\epsilon$. Together with Lemma 4.2 this yields the desired result. (b) The vector $x$ with $x_{j^{*}+1}=\ldots=x_{n}=1, x_{n+1}=1$, and $x_{j}=0$ for all other $j$, is feasible, because

$$
\sum_{j=j^{*}+1}^{n} w_{j}+w_{n+1} \leq \Delta+w_{n+1} \leq 2 \epsilon(1+\kappa)+1+\kappa=(1+\kappa)(1+2 \epsilon) \leq 2,
$$

by definition of $\epsilon$. Hence, we must have $\sum_{j=j^{*}+1}^{n} p_{j}+p_{n+1}<1+\epsilon$ and we conclude as in (a).

Corollary $4.4 p_{j}^{*} \geq 1-5 \epsilon$ and $w_{j^{*}}+w_{n+1}>2$.

Proof. Lemma 4.2 (b) and Lemma 4.3 yield the bound on $p_{j^{*}}$. If $w_{j^{*}}+w_{n+1} \leq 2$ then

$$
\frac{v^{*}}{v^{Z}} \leq \frac{2}{2-6 \epsilon}<2-\epsilon
$$

a contradiction.

Lemma 4.5 (a) $w_{n+1}>1-\epsilon$. (b) $w_{j^{*}}<1+3 \epsilon$. 
Proof. (a) By our indexing of variables in non-increasing order of values $p_{j} / w_{j}$,

$$
\frac{p_{1}+\ldots+p_{n}}{w_{1}+\ldots w_{n}} \geq \frac{p_{n+1}}{w_{n+1}}
$$

and thus, since $b=2$,

$$
w_{n+1} \geq \frac{1-\epsilon}{1+\epsilon}\left(w_{1}+\ldots+w_{n}\right)>\frac{1-\epsilon}{1+\epsilon}\left(2-w_{n+1}\right),
$$

from which the result follows.

(b) This follows from

$$
w_{j^{*}} \leq w_{1}+\ldots+w_{n}=2-x_{n+1}^{*} w_{n+1}<2-(1-2 \epsilon)(1-\epsilon)<1+3 \epsilon .
$$

Define

$$
P=\left\{1 \leq j \leq N: j>n+1 \text { and } w_{j}+w_{j^{*}} \leq 2\right\} .
$$

In what follows we consider an arbitrary vector $\hat{x}$ that satisfies $\sum_{j} w_{j} \hat{x}_{j} \leq b$, all clique inequalities, and $0 \leq \hat{x}_{j} \leq 1, \forall j$. We will assume that $\sum_{j} p_{j} \hat{x}_{j}>(2-\epsilon) v^{Z}$, and show that this leads to a contradiction, thereby proving Theorem 1.3.

Lemma $4.6 \sum_{j \in P} \hat{x}_{j} p_{j} \leq 60 \epsilon$.

Proof. Assume by contradiction that $\sum_{j \in P} \hat{x}_{j} p_{j}>60 \epsilon$. The proof will construct a subset $A \subseteq P$ such that

$$
\begin{aligned}
& w_{j^{*}}+\sum_{j \in A} w_{j} \leq 2, \text { and } \\
& \sum_{j \in A} p_{j}>6 \epsilon .
\end{aligned}
$$

This will provide a contradiction since in that case $v^{Z}>1+\epsilon$ and

$$
\frac{v^{\omega}}{v^{Z}} \leq \frac{2}{1+\epsilon}<2-\epsilon
$$

Since $\sum_{j \in P} w_{j} \hat{x}_{j} \leq 2-w_{j^{*}} \hat{x}_{j^{*}}$, it follows that there exists $T \subseteq P$ with

$$
\begin{aligned}
& \sum_{j \in T} w_{j} \leq 2-w_{j^{*}} \hat{x}_{j^{*}}, \text { and } \\
& \sum_{j \in T} p_{j}>30 \epsilon .
\end{aligned}
$$

Let $\alpha, \beta$ be defined as follows. If $w_{j^{*}} \leq 1$, then $\alpha=\beta$, whereas if $w_{j^{*}}>1$ then $\alpha=1-\left(w_{j^{*}}-1\right)$ and $\beta=w_{j^{*}}$. Note that in either case $\alpha \leq \beta, \beta-\alpha<6 \epsilon$ (by Lemma 4.5(b)), $\beta-\alpha<\alpha \leq 2-\beta$, and $w_{j^{*}}+2-\beta=2$. Let

$$
T=\{i(1), i(2), \cdots, i(|T|)\} .
$$

Consider a family of closed intervals $I_{k}=\left[a_{k}, b_{k}\right], k=1, \cdots,|T|$, where

$$
\begin{aligned}
& a_{1}=0, \\
& b_{k}=\sum_{j=1}^{k} w_{i(j)}, \quad k=1, \cdots,|T|, \\
& a_{k+1}=b_{k}, \quad k=1, \cdots,|T|-1 .
\end{aligned}
$$

Then we can partition the intervals $I_{k}$ into at most 5 disjoint classes (some of which may be empty), 
(1) Intervals $I_{k}$ with $b_{k} \leq \alpha$,

(2) Intervals $I_{k}$ with $a_{k} \geq \beta$,

(3) Intervals $I_{k}$ with $a_{k}>\alpha$ and $b_{k}<\beta$, and

(4) At most two other intervals (each constituting a class).

Let $A$ be the class with largest sum of $p_{j}$ - thus $A$ satisfies (113). By definition of $P$ and $T$, by Lemma (4.5)(b) and by construction of the $I_{k}, A$ satisfies (112).

Lemma 4.3 and Lemma 4.6 imply that

$$
\sum_{j=1, j \neq j^{*}}^{n} \hat{x}_{j} p_{j}+\sum_{j \in P} \hat{x}_{j} p_{j} \leq 64 \epsilon,
$$

In the rest of this Section we will show that the remaining terms in $\sum_{j} \hat{x}_{j} p_{j}$ amount to less than $(3 / 2+4 \epsilon) v^{Z}$. Thus, overall

$$
\sum_{j} \hat{x}_{j} p_{j} \leq 64 \epsilon+(3 / 2+4 \epsilon) v^{Z} \leq(3 / 2+68 \epsilon) v^{Z} \leq(2-\epsilon) v^{Z}
$$

(by our choice of $\epsilon$ ) which is the desired contradiction. Note that the remaining terms consist of

- index $j^{*}$, and

- indices $j \neq j^{*}$ with $w_{j}+w_{j^{*}}>2$. Let $\mathcal{I}$ be the set of such indices $j$.

Our approach will be to upper-bound the sum of remaining terms by the value of a linear program, whose constraints will primarily amount to clique inequalities, restricted to variables $x_{j}$ with $j \in \mathcal{I} \cup\left\{j^{*}\right\}$.

We partition $\mathcal{I}$ into

$$
\mathcal{S}=\left\{j \in \mathcal{I}: w_{j} \leq 1\right\} \text { and } \mathcal{L}=\left\{j \in \mathcal{I}: w_{j}>1\right\} .
$$

Lemma 4.7 Suppose $\mathcal{S}=\emptyset$. Then $\hat{x}_{j^{*}} p_{j^{*}}+\sum_{j \in \mathcal{I}} \hat{x}_{j} p_{j} \leq 1+\epsilon$.

Proof. By definition of $\mathcal{I}$ we have that

$$
\sum_{j \in \mathcal{I} \cup\left\{j^{*}\right\}} x_{j} \leq 1
$$

is a clique inequality, and the result follows by Lemma $4.2(\mathrm{a})$.

The remainder of the proof handles the case $\mathcal{S} \neq \emptyset$, and consequently, by definition of $\mathcal{I}, w_{j^{*}}>1$. Note that for each $j \in \mathcal{I}$ we have $j>n$ and $w_{j}>1-3 \epsilon$ (this by Lemma 4.5(b)). Thus, since $\sum_{j=1}^{N} w_{j} \hat{x}_{j} \leq 2$, we also have

$$
\sum_{j \in \mathcal{I} \cup\left\{j^{*}\right\}} \hat{x}_{j} \leq 2+4 \epsilon .
$$

Also note that if $j \in \mathcal{S}$,

$$
p_{j} \leq p_{j} / w_{j} \leq p_{j^{*}} / w_{j^{*}}<p_{j^{*}} .
$$

\section{Definition:}


- $s(1)=\operatorname{argmax}\left\{p_{j}: j \in \mathcal{S}\right\}$,

- $\mathcal{L}^{1}=\left\{j \in \mathcal{L}: w_{j}+w_{s(1)}>2\right\}$, and

- $\mathcal{L}^{2}=\mathcal{L}-\mathcal{L}^{1}$.

Lemma 4.8 Suppose $|\mathcal{S}|=1$. Then $\hat{x}_{j^{*}} p_{j^{*}}+\sum_{j \in \mathcal{I}} \hat{x}_{j} p_{j} \leq 1+\epsilon$.

Proof. Let $\mathcal{S}=\{i\}$. Then $\mathcal{L}^{1}=\left\{j \in \mathcal{L}: w_{i}+w_{j}>2\right\}$. The following are clique inequalities:

$$
\begin{aligned}
& x_{j^{*}}+\sum_{j \in \mathcal{L}^{1}} x_{j}+\sum_{j \in \mathcal{L}^{2}} x_{j} \leq 1, \\
& x_{j^{*}}+\sum_{j \in \mathcal{L}^{1}} x_{j}+x_{i} \leq 1,
\end{aligned}
$$

and thus, $\hat{x}_{j^{*}} p_{j^{*}}+\hat{x}_{i} p_{i}+\sum_{j \in \mathcal{L}} \hat{x}_{j} p_{j}$ is upper-bounded by the value of the linear program

$$
\max \left\{p_{j^{*}} x_{j^{*}}+p_{i} x_{i}+\sum_{j \in \mathcal{L}} p_{j} x_{j}: \text { s.t. (122)-(123), each variable in }[0,1]\right\} .
$$

We conclude that

$$
\hat{x}_{j^{*}} p_{j^{*}}+\hat{x}_{i} p_{i}+\sum_{j \in \mathcal{L}} \hat{x}_{j} p_{j} \leq \max \left\{\max _{k \in \mathcal{L}^{1} \cup\left\{j^{*}\right\}} w_{k}, w_{i}+\max _{k \in \mathcal{L}^{2}}\left\{w_{k}\right\}\right\} \leq v^{Z},
$$

where the last inequality follows because by definition of $\mathcal{L}^{2}$, there is an integer feasible solution to $\mathcal{K} \mathcal{M} \mathcal{A} \mathcal{X}$ of value precisely $w_{i}+\max _{k \in \mathcal{L}^{2}}\left\{w_{k}\right\}$, and there is one of $\max _{k \in \mathcal{L}^{1} \cup\left\{j^{*}\right\}} w_{k}$.

In the remainder of the proof we will assume $|\mathcal{S}| \geq 2$. Consider the linear program

$$
\theta=\max \sum_{j \in \mathcal{L}^{1} \cup\left\{j^{*}\right\}} p_{j} x_{j}+\sum_{j \in \mathcal{L}^{2}} p_{j} x_{j}+\sum_{j \in \mathcal{S}} p_{j} x_{j}
$$

Subject to:

$$
\begin{array}{ll}
(\alpha): & \sum_{j \in \mathcal{L}^{1} \cup\left\{j^{*}\right\}} x_{j}+\sum_{j \in \mathcal{L}^{2}} x_{j} \leq 1, \\
(\beta): & \sum_{j \in \mathcal{L}^{1} \cup\left\{j^{*}\right\}} x_{j}+x_{s(1)} \leq 1, \\
(\gamma): & \sum_{j \in \mathcal{L}^{1} \cup\left\{j^{*}\right\}} x_{j}+\sum_{j \in \mathcal{L}^{2}} x_{j}+\sum_{j \in \mathcal{S}} x_{j} \leq 2+4 \epsilon, \\
& x \geq 0 .
\end{array}
$$

Here, (127) and (128) are clique inequalities, and (129) is the same as (120). Thus,

$$
\sum_{j \in \mathcal{L} \cup j^{*}} \hat{x}_{j} p_{j}+\sum_{j \in \mathcal{S}} \hat{x}_{j} p_{j} \leq \theta .
$$

In the above formulation, we have indicated the names of the dual variables. Next, define: 
- $h=\operatorname{argmax}\left\{p_{j}: j \in \mathcal{L}^{1} \cup\left\{j^{*}\right\}\right\}$.

- $s(2)=\operatorname{argmax}\left\{p_{j}: j \in \mathcal{S}-s(1)\right\}$.

Lemma 4.9 Suppose $\left\{j \in \mathcal{L}: p_{j}>p_{s(2)}\right\} \subseteq \mathcal{L}^{1}$. Then $\theta \leq(3 / 2+2 \epsilon) v^{Z}$.

Proof. By construction (and (121)), $p_{h}>p_{s(1)} \geq p_{s(2)} \geq p_{j}$ for each $j \in \mathcal{L}^{2}$. Thus, the following vector is a dual feasible solution to the LP (126)-(130):

$$
\alpha=0, \quad \beta=p_{h}-\frac{p_{s(1)}+p_{s(2)}}{2}, \quad \gamma=\frac{p_{s(1)}+p_{s(2)}}{2} .
$$

The value of this dual feasible solution is

$$
p_{h}+(1+4 \epsilon) \frac{p_{s(1)}+p_{s(2)}}{2} \leq\left(\frac{3}{2}+2 \epsilon\right) \max \left\{p_{h}, p_{s(1)}+p_{s(2)}\right\} .
$$

This concludes the proof, since we have an integer feasible solution to $\mathcal{K} \mathcal{M} \mathcal{A} \mathcal{X}$ by setting $x_{h}=1$ (and all other $x_{j}=0$ ), and another by setting $x_{s(1)}=x_{s(2)}=1$ and all other $x_{j}=0$.

Lemma 4.10 Suppose there exists $k \in \mathcal{L}^{2}$ with $p_{k}>p_{s(2)}$. Then $\theta \leq(3 / 2+2 \epsilon) v^{Z}$.

Proof. Without loss of generality we can assume $k=\operatorname{argmax}\left\{p_{j}: j \in \mathcal{L}^{2}\right\}$. As previously, $p_{h}>p_{s(1)}$. Also, since $k \in \mathcal{L}, w_{k}>1$. Since $\sum_{j=1}^{n} w_{j} \leq 2$, and $w_{j^{*}}>1$, we therefore have $k>n$ and so

$$
p_{k} / w_{k} \leq p_{j^{*}} / w_{j^{*}}
$$

Further, $s(1) \in \mathcal{I}$ implies $w_{j^{*}}+w_{s(1)}>2$. But since $k \in \mathcal{L}^{2}, w_{k}+w_{s(1)} \leq 2$. Consequently, $w_{j^{*}}>w_{k}$, and using (133) we have

$$
p_{k}<p_{j^{*}} \leq p_{h}
$$

As a result, if $p_{k} \leq p_{s(1)}$, the following is a dual feasible solution to the LP (126)-(130):

$$
\alpha=0, \quad \beta=p_{h}-\frac{p_{s(1)}+p_{k}}{2}, \quad \gamma=\frac{p_{s(1)}+p_{k}}{2} ;
$$

and if $p_{k}>p_{s(1)}$, the following vector is dual feasible:

$$
\alpha=p_{h}-\frac{p_{s(1)}+p_{k}}{2}, \quad \beta=0, \quad \gamma=\frac{p_{s(1)}+p_{k}}{2} .
$$

In either case, the value of the solution is

$$
p_{h}+(1+4 \epsilon) \frac{p_{s(1)}+p_{k}}{2} \leq\left(\frac{3}{2}+4 \epsilon\right) \max \left\{p_{h}, p_{s(1)}+p_{k}\right\}
$$

This concludes the proof, since we have an integer feasible solution to $\mathcal{K} \mathcal{M} \mathcal{A} \mathcal{X}$ by setting $x_{h}=1$ (and all other $x_{j}=0$ ), and another by setting $x_{s(1)}=x_{k}=1$ and all other $x_{j}=0$.

Acknowledgment. I thank Bruce Shepherd for suggesting the questions in Section 4 during a visit to McGill University which was supported by his NSERC grant. 


\section{References}

[1] E. BAlas, Disjunctive Programs: Cutting Planes from Logical Conditions, in O.L. Mangasarian et al., eds., Nonlinear Programming 2 (1975) Academic Press, 279 - 312.

[2] E. Balas, S. Ceria and G. CornuéJols, A lift-and-project cutting plane algorithm for mixed 0-1 programs, Mathematical Programming 58 (1993), 295 - 324.

[3] D. Bienstock, Approximate formulations for 0-1 knapsack sets, Operations Research Letters 36 (2008), 317-320.

[4] D. Bienstock And N.ÖzBAy, Tree-width and the Sherali-Adams operator, Discrete Optimization 1 (2004) 13-22.

[5] D. Bienstock and M. Zuckerberg, Subset algebra lift operators for 0-1 Integer Programming, SIAM J. Optimization 15 (2004) 63-95.

[6] D. Bienstock And M. Zuckerberg, Approximate fixed-rank closures of covering problems Math. Programming 105 (2006), $9-27$.

[7] T. Carnes and D. Shmoys, Primal-Dual Schema for Capacitated Covering Problems, Proc. IPCO 2008.

[8] R.D. Carr, L.K. Fleischer, V.J. Leung, C.A. Phillips, Strengthening Integrality Gaps for Capacitated Network Design and Covering Problems, Proc. 2000 SODA,

[9] W. Cook And S. Dash, On the matrix-cut rank of polyhedra, Mathematics of Operations Research 26 (2001), 19 - 30.

[10] M.X. Goemans And L. Tunçel, When does the positive semidefiniteness constraint help in lifting procedures?, Mathematics of Operations Research 26 (2001), 796 - 815.

[11] Z. Gu, G. Nemhauser and M. Savelsbergh, Lifted flow cover inequalities for mixed 0/1 integer programs, Math. Programming 85 (1999), 439 -467.

[12] A.N. Letchford, On disjunctive cuts for combinatorial optimization, J. of Comb. Opt. 5 (2001) $299-315$.

[13] G.L. Nemhauser and L.A. Wolsey, Integer and Combinatorial Optimization, Wiley, New York (1988).

[14] M. Padberg, T. Van Roy, and L. Wolsey, Valid linear inequalities for fixed charge problems, Operations Research 33 (1985), 842 - 861.

[15] N. Robertson and P.D. Seymour, Graph Minors II: Algorithmic aspects of tree-width, J. Algorithms 7 (1986), 309-322.

[16] S. Sherali And W. Adams, A hierarchy of relaxations between the continuous and convex hull representations for zero-one programming problems, SIAM J. on Discrete Mathematics 3 (1990), $411-430$.

[17] M. Van Vyve and L.A. Wolsey, Approximate extended formulations, Math. Program. 105 (2006) $501-522$. 
[18] M. Zuckerberg, A Set Theoretic Approach to Lifting Procedures for 0,1 Integer Programming PhD dissertation, Columbia University (2003). 